\title{
Competition between Granule Bound Starch Synthase and Starch Branching Enzyme in Starch Biosynthesis
}

Huaxin Han ${ }^{1,2}$, Chuantian Yang ${ }^{1,2}$, Jihui Zhu ${ }^{1,2}$, Lixia Zhang ${ }^{3}$, Yeming Bai ${ }^{4}$, Enpeng Li $i^{12^{*}}$ (D) and Robert G. Gilbert ${ }^{1,24^{*}}$

\begin{abstract}
Background: Starch branching enzymes (SBE) and granule-bound starch synthase (GBSS) are two important enzymes for starch biosynthesis. SBE mainly contributes to the formation of side branches, and GBSS mainly contributes for the synthesis of amylose molecules. However, there are still gaps in the understanding of possible interactions between SBE and GBSS.
\end{abstract}

Results: Nineteen natural rice varieties with amylose contents up to $28 \%$ were used. The molecular structure, in the form of the chain-length distribution (CLDs, the distribution of the number of monomer units in each branch) was measured after enzymatic debranching, using fluorophore-assisted carbohydrate electrophoresis for amylopectin and size- exclusion chromatography for amylose. The resulting distributions were fitted to two mathematical models based on the underlying biosynthetic processes, which express the CLDs in terms of parameters reflecting relevant enzyme activities.

Conclusions: Finding statistically valid correlations between the values of these parameters showed that GBSSI and SBEI compete for substrates during rice starch biosynthesis, and synthesis of amylose short chains involves several enzymes including GBSSI, SBE and SSS (soluble starch synthase). Since the amylose CLD is important for a number of functional properties such as digestion rate, this knowledge is potentially useful for developing varieties with improved functional properties.

Keywords: Starch, Biosynthesis, Enzyme actions, Rice, Structural characterization

\section{Background}

Amylose and amylopectin are the two main components of starch, which is a complex branched glucose polymer. Amylose has moderate molecular weight with a small number of long-chain branches, and amylopectin has large molecular weight with a vast number of short-chain branches (Chiaramonte et al. 2012; Tester et al. 2004).

Concerted actions of a series of biosynthetic enzymes with multiple isoforms, mainly starch synthases (SS), starch branching enzymes (SBE), starch debranching enzymes (DBE) and ADP-glucose pyrophosphorylase polypeptide (AGPase), are involved in starch biosynthesis in

\footnotetext{
* Correspondence: lep@yzu.edu.cn; b.gilbert@uq.edu.au

${ }^{1}$ Jiangsu Key Laboratory of Crop Genetics and Physiology, Key Laboratory of

Plant Functional Genomics of the Ministry of Education, Jiangsu Key

Laboratory of Crop Genetics and Physiology, College of Agriculture,

Yangzhou University, Yangzhou 225009, People's Republic of China

Full list of author information is available at the end of the article
}

cereal endosperms (Akihiro et al. 2005; HennenBierwagen et al. 2008). Granule-bound starch synthase I (GBSSI) is the key enzyme for amylose biosynthesis, predominantly elongating the amylose chains; the soluble starch synthases (SSS) are mainly responsible for amylopectin elongation (Ball et al. 1998; Denyer et al. 2001; Wang et al. 2015). Starch branches are formed by starch branching enzymes (SBE) through transferring an oligosaccharide fragment with a non-reducing end and a $(1 \rightarrow 6)-\alpha$ glycosidic bond formed by the C6 end of glucose in the glycoside chain (Satoh et al. 2003; Zeeman et al. 2010; Tanaka et al. 2004). Although SBE and SS are mainly involved in amylopectin synthesis, recent studies show that they also might be involved in amylose synthesis ( $\mathrm{Li}$ et al. 2015; Yu et al. 2018). However, little is known about the details of how the individual isoforms of SBE and SS contribute to the amylose and amylopectin chain-length distribution 
(CLD) (Li et al. 2017; Li et al. 2015; Guan and Preiss 1993). One isoform of the starch debranching enzymes (DBEs) hydrolyzes widely-spaced $(1 \rightarrow 6)-\alpha$ bonds of the polysaccharide chain to achieve spacing between amylopectin branch points such that crystallization between amylopectin chains can occur (Nakamura et al. 1997). AGPase catalyzes glucose-1-phosphate and ATP to form pyrophosphate and ADPG (ADP-glucose). ADPG is the initial glucose-based donor of starch biosynthesis and a substrate for starch synthesis, whose concentration directly affects the rate and efficiency of starch synthesis (Baroja-Fernandez et al. 2012; Bowsher et al. 2007).

The present study looks for the presence of concerted actions between enzymes, involved in the synthesis of both amylopectin and amylose by investigating 19 natural rice samples (Table 1). The range of amylose, up to $28 \%$, goes to significantly higher values than normally encountered in rice. Enzyme actions are investigated through studying the chain-length distributions (CLDs: the number or weight distributions of monomer units in individual chains), these being measured by enzymatically debranching the starch and determining the molecular weight distributions of the resulting linear polymers by fluorophoreassisted carbohydrate electrophoresis (FACE) for amylopectin chains and size-exclusion chromatography (SEC, a type of gel-permeation chromatography, GPC) for amylose chains. One of the novel aspects of the present study is to parameterize these CLDs using biosynthesis-based

Table 1 Rice samples from different botanical sources

\begin{tabular}{lll}
\hline Number & Name & Country of origin \\
\hline 1 & IRTP 19771-G1 & Ivory Coast \\
2 & GERVEX 1686-C1 & Greece \\
3 & IRGC 64858-1 & South Korea \\
4 & Qingsiai 16B & China \\
5 & IRGC 12872-1 & New Zealand \\
6 & UPR 191-66 & India \\
7 & IRGC 62683-1 & China \\
8 & MONOLAYA & United States \\
9 & GERVEX 1638-C1 & United States \\
10 & K24 & Uganda \\
11 & NERICA-L & Africa \\
12 & IRGC 70371-1 & China \\
13 & IRGC 28036-1 & Pakistan \\
14 & IRGC 46659-1 & India \\
15 & IRGC 53437-1 & China \\
16 & Yunlu 103 & China \\
17 & IRGC 38606-1 & India \\
18 & IRGC 3272-1 & Argentina \\
19 & Wuxiangjing 14 & China \\
\hline & & \\
\hline & &
\end{tabular}

models, which provide a very good fit to these data. The result is a comparatively small set of parameter values, representing various biosynthesis enzyme activities, which can then be used to find statistically valid correlations among themselves, thereby revealing interactions between SBE, DBE, SS, and GBSS.

\section{Results and Discussion}

\section{Fitting Parameters}

The amylopectin CLDs are shown in Fig. 1, normalized to the highest peak. All 19 samples show typical amylopectin distributions, and three regions can be seen. The amylopectin model fitting parameters are shown in Table 2 with the fits shown in the Supporting Information (Additional file 1: Figure S1-a and S1-b). Amylopectin chains are grouped into $\mathrm{A}, \mathrm{B}$ and $\mathrm{C}$ chain, and $\mathrm{B}$ chain can be further divided into B1, B2, B3 chains (Tester et al. 2004). In the amylopectin fitting model, region 1 can be generally considered representing $\mathrm{A}$ and B1 chains, region 2 represents B2 chains and region 3 represents B3 chains. As detailed in the methods sections, the biosynthesis parameters (which are the ratio of activities of branching and synthase enymes) $\beta_{(\mathrm{i})}$ and $\beta_{(\mathrm{ii})}$ are calculated based on CLDs of region1, $\beta_{(\mathrm{iii})}$ and $\beta_{(\mathrm{iv})}$ are calculated based on those of region 2 , and $\beta_{(\mathrm{v})}$ and $\beta_{(\mathrm{vi})}$ on those of region 3 . For the biosynthesis parameters representing the relative rates of chain elongation, $h_{(\mathrm{iii} / \mathrm{i})}$ is the ratio of region 2 height to region 1 height, while $h_{(\mathrm{v} / \mathrm{i})}$ is the ration of region 3 height to region 1 height.

The weight CLDs of amylopectin and amylose from SEC are given in Fig. 2, normalized to the highest peak (which is for short amylopectin chains). One normally divides CLDs into amylose and amylopectin portions where the CLD shows a minimum, which is almost always close to degree of polymerization (abbreviation DP, given the symbol $X$ ) close to 100 . Those for $\lesssim 100$ are amylopectin chains and those with $X \gtrsim 100$ are amylose chains. While SEC is needed for the amylose CLDs, the SEC amylopectin CLDs are not used here because FACE can give a much better resolution for DP $\lesssim 180$. Amylose CLDs were fitted with the biosynthesis-based models described previously (Wu et al. 2013a; Yu et al. 2019; Nada et al. 2017). The final outcomes of these models is that various features in different regions of the CLDs can be expressed in terms of just two parameters: $\beta$ (with appropriate subscripts for amylopectin or amylose and for which feature), which is the ratio of the activities of the starch branching (SBE) and starch synthase (SS) dominating the CLD in that feature, and $h$ (again with appropriate subscripts) which is the relative activity of the dominant SS in that region (Additional file 1: Figure S1-c and S1-d). The amylose fitting parameters are shown in Table 3. 




Fig. 1 CLDs from FACE analysis, normalized to the highest amylopectin peak

\section{Correlations between Parameters for Amylopectin and} Amylose

It is noted that the complete chain-length distribution can be used to calculate the degree of branching through the formula (Wu et al. 2013b): DB $=\frac{\sum_{X} N_{d e}(X)}{\sum_{X} X N_{d e}(X)}$, which is an exact result arising from the definition of the degree of branching and so this quantity is not an independent structural variable, given a complete parameterization of the CLD, as done here. However, it is a useful and commonly used property, so correlations with the independent structural are listed here. The correlations between amylopectin and amylose structural parameters are listed in Table 4 . It is seen that $\beta_{\mathrm{Am} \text {,(iii) }}$ significantly negatively correlates with $\beta_{(\mathrm{iii})}$, and strongly negatively correlates with $\beta_{(\mathrm{v})}$. $\beta_{\mathrm{Am} \text {,(iii) }}$ represents long amylose chains, and $\beta_{(\mathrm{iii})}$ and $\beta_{(\mathrm{v})}$ represent intermediate and long amylopectin chains. This suggests that the synthesis of long

Table 2 Parameter values from fitting the biosynthesis model to the amylopectin CLDs of the 19 rice samples

\begin{tabular}{lllllllll}
\hline Sample & $\beta_{(\mathrm{i})} / 10^{-3}$ & $\beta_{(\mathrm{ii})} / 10^{-3}$ & $\beta_{(\mathrm{iii})} / 10^{-3}$ & $\beta_{(\mathrm{iv})} / 10^{-3}$ & $\beta_{(\mathrm{v})} / 10^{-3}$ & $\beta_{(\mathrm{vi})} / 10^{-3}$ & $h_{(\mathrm{iii} / \mathrm{i})} / 10^{-3}$ & $h_{(\mathrm{v} / \mathrm{i})} / 10^{-3}$ \\
\hline 1 & $113.0 \pm 0.1$ & $28.2 \pm 0.7$ & $57.5 \pm 0.3$ & $14.7 \pm 0.2$ & $44.7 \pm 0.1$ & $13.5 \pm 0.2$ & $104.2 \pm 0.3$ & $7.4 \pm 0.1$ \\
2 & $113.5 \pm 2.1$ & $33.0 \pm 1.6$ & $60.9 \pm 1.8$ & $12.9 \pm 0.3$ & $46.0 \pm 4.7$ & $10.0 \pm 1.9$ & $111.1 \pm 5.3$ & $7.7 \pm 0.6$ \\
3 & $97.3 \pm 0.7$ & $29.8 \pm 0.4$ & $54.6 \pm 0.1$ & $14.1 \pm 0.6$ & $43.3 \pm 1.0$ & $10.8 \pm 0.3$ & $95.8 \pm 2.0$ & $5.8 \pm 0.2$ \\
4 & $117.0 \pm 1.3$ & $26.7 \pm 0.5$ & $61.3 \pm 2.2$ & $13.6 \pm 1.8$ & $43.7 \pm 1.1$ & $12.6 \pm 0.7$ & $111.6 \pm 2.6$ & $7.7 \pm 0.2$ \\
5 & $100.1 \pm 1.3$ & $33.4 \pm 0.8$ & $55.6 \pm 0.3$ & $16.2 \pm 0$ & $49.4 \pm 0.6$ & $15.5 \pm 1.9$ & $99.8 \pm 3.2$ & $6.7 \pm 0.3$ \\
6 & $97.4 \pm 0.2$ & $27.0 \pm 1.9$ & $53.7 \pm 0.1$ & $14.4 \pm 0.2$ & $54.6 \pm 0.5$ & $8.5 \pm 0.3$ & $105.6 \pm 1.6$ & $10.5 \pm 1.1$ \\
7 & $97.4 \pm 6.5$ & $37.3 \pm 3.8$ & $53.7 \pm 2.4$ & $18.3 \pm 0.9$ & $43.2 \pm 4.2$ & $21.1 \pm 1.5$ & $115.5 \pm 2.8$ & $7.8 \pm 0.3$ \\
8 & $119.6 \pm 0.8$ & $23.2 \pm 0.4$ & $56.8 \pm 0.7$ & $17.4 \pm 0.0$ & $40.8 \pm 0.8$ & $13.5 \pm 1.6$ & $103.9 \pm 1.6$ & $8.1 \pm 0.5$ \\
9 & $123.0 \pm 0.6$ & $21.4 \pm 0.4$ & $53.3 \pm 0.7$ & $21.4 \pm 0.1$ & $37.8 \pm 4.5$ & $12.7 \pm 1.4$ & $94.1 \pm 0.2$ & $7.1 \pm 0.2$ \\
10 & $121.5 \pm 0.2$ & $26.1 \pm 2.1$ & $55.8 \pm 0.2$ & $17.9 \pm 0.3$ & $39.4 \pm 1.5$ & $6.9 \pm 0.5$ & $107.7 \pm 1.9$ & $8.0 \pm 0.2$ \\
11 & $118.2 \pm 2.4$ & $25.0 \pm 1.0$ & $52.7 \pm 2.9$ & $21.0 \pm 2.2$ & $37.8 \pm 3.1$ & $15.4 \pm 2.2$ & $99.1 \pm 1.9$ & $6.8 \pm 0.4$ \\
12 & $123.5 \pm 0.9$ & $19.2 \pm 0.7$ & $57.0 \pm 4.3$ & $13.4 \pm 2.9$ & $35.9 \pm 1.5$ & $17.6 \pm 1.0$ & $88.6 \pm 0.2$ & $6.0 \pm 0.4$ \\
13 & $115.2 \pm 3.9$ & $32.7 \pm 1.5$ & $54.2 \pm 0.6$ & $17.2 \pm 0.2$ & $37.4 \pm 0.7$ & $13.7 \pm 0.8$ & $96.9 \pm 5.5$ & $6.9 \pm 0.6$ \\
14 & $106.2 \pm 0.7$ & $25.8 \pm 0.9$ & $52.0 \pm 0.7$ & $18.0 \pm 0.4$ & $35.2 \pm 1.2$ & $7.1 \pm 0.3$ & $97.7 \pm 5.5$ & $7.3 \pm 0.3$ \\
15 & $103.8 \pm 0.1$ & $27.9 \pm 2.6$ & $50.4 \pm 3.1$ & $18.3 \pm 1.7$ & $38.1 \pm 5.2$ & $11.6 \pm 1.1$ & $82.3 \pm 2.0$ & $6.1 \pm 0.3$ \\
16 & $122.5 \pm 0.5$ & $15.9 \pm 1.3$ & $54.5 \pm 0.8$ & $15.0 \pm 3.8$ & $32.9 \pm 4.9$ & $17.3 \pm 2.3$ & $102.6 \pm 1.6$ & $9.3 \pm 0.1$ \\
17 & $110.9 \pm 0.2$ & $32.7 \pm 3.2$ & $51.7 \pm 4.3$ & $18.4 \pm 2.8$ & $31.1 \pm 0.2$ & $16.8 \pm 2.9$ & $93.3 \pm 2.7$ & $6.6 \pm 0.3$ \\
18 & $115.6 \pm 1.6$ & $35.1 \pm 0.7$ & $50.2 \pm 0.3$ & $19.3 \pm 0.6$ & $41.1 \pm 3.8$ & $9.5 \pm 1.1$ & $104.5 \pm 2.1$ & $6.6 \pm 0.1$ \\
19 & $111.2 \pm 1.7$ & $30.3 \pm 1.1$ & $49.6 \pm 1.3$ & $18.2 \pm 0.6$ & $38.8 \pm 2.1$ & $9.8 \pm 0.4$ & $105.6 \pm 4.1$ & $6.1 \pm 0.5$ \\
\hline & & & & & & & &
\end{tabular}



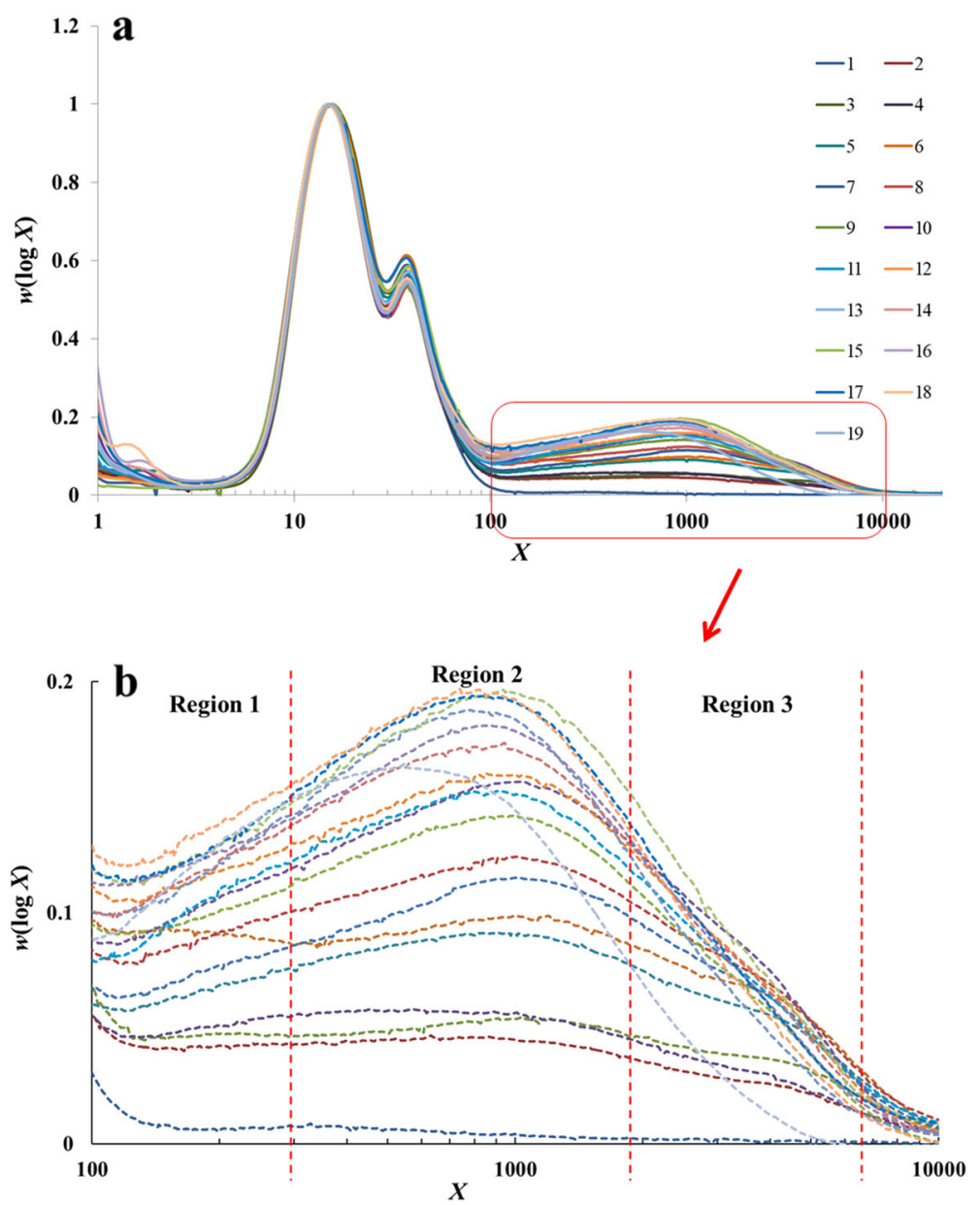

Fig. 2 SEC weight CLDs of the whole range of debranched starch branches. All distributions are normalized to the highest amylopectin peak

amylose chains competes with the syntheses of intermediate and long amylopectin chains, especially the long amylopectin chains, and perhaps these two syntheses could happen at the same site in starch granules. It has been shown that GBSSI is the dominant enzyme controlling long amylose chain synthesis ( $\mathrm{Li}$ et al. 2015; Wang et al. 2015). SSIII is believed to be the main SS and SBEI the main SBE involved in the synthesis of longer amylopectin chains (DP > 25) (Bowsher et al. 2007; Zhang et al. 2008). It is seen that $h_{(\mathrm{iii} / \mathrm{i})}$ and $h_{(\mathrm{v} / \mathrm{i})}$ have no signifi-

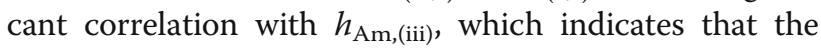
interaction between SSIII and GBSSI is not significant. SBEI can transfer long glucosyl chains $(D P>20)$ to form long amylopectin branches, while GBSSI can elongate amylose chains with long glucosyl chains $(D P>20)$ (Denyer et al. 2001; Wang et al. 2015). The correlation results suggest that both GBSSI and SBEI compete for the similar substrates (long glucosyl chains) during starch biosynthesis, an inference not previously reported. The correlation result also shows that $\beta_{\mathrm{Am} \text {,(iii) }}$ is not significantly correlates with $\beta_{(\mathrm{i})}$ and $\beta_{(\mathrm{ii})}$, which shows that
GBSSI has little interaction with SBEIIb in rice. Future experiments are needed to analyze the activity of different enzyme isoforms (especially GBSSI and SBEI) and the interactions between different isoforms.

Interestingly, there is no correlation observed between $\beta_{\mathrm{Am} \text {, (i) }}$ and $\beta_{\mathrm{Am} \text {,(ii) }}$ and amylopectin structural parameters. Earlier studies have shown that, besides GBSSI, some branching enzymes and soluble starch synthases are also involved in short amylose chain biosynthesis (Buleon et al. 1998; Li et al. 2015). This might mean that the synthesis of short amylose chains involves more enzymes than those for long amylopectin chains, which could explain why the structural parameters of short amylose chains showed no significant correlation with those of amylopectin.

It is seen that $\beta_{(\mathrm{iii})}$ and $\beta_{(\mathrm{v})}$ show negative correlation with $h_{\mathrm{Am} \text {,(i,) }} h_{\mathrm{Am} \text {,(ii) }}$ and $h_{\mathrm{Am} \text {,(iii) }}$. The amylose $h$ parameters are of course strongly positively correlated with amylose content (Table 4). GBSSI is believed to play a key role in determining the amylose content of cereal grains, and it competes with SBEI during starch 
Table 3 Parameter values from fitting the biosynthesis model to the amylose CLDs

\begin{tabular}{|c|c|c|c|c|c|c|c|}
\hline Sample & Amylose content / \% & $\beta_{\text {Am, (i) }} / 10^{-3}$ & $\beta_{\mathrm{Am},(\mathrm{ii})} / 10^{-3}$ & $\beta_{\mathrm{Am}, \text { (iii) }} / 10^{-3}$ & $h_{\mathrm{Am}, \text { (i) }} / 10^{-3}$ & $h_{\mathrm{Am} \text {,(ii) }} / 10^{-3}$ & $h_{\mathrm{Am}, \text { (iii) }} / 10^{-3}$ \\
\hline 1 & $1.1 \pm 0.7$ & N/A & N/A & N/A & N/A & N/A & N/A \\
\hline 2 & $9.6 \pm 0.9$ & $11.1 \pm 0.0$ & $2.6 \pm 0.0$ & $0.6 \pm 0.0$ & $20.4 \pm 0.0$ & $23.9 \pm 0.2$ & $21.8 \pm 2.6$ \\
\hline 3 & $10.9 \pm 0.2$ & $10.7 \pm 0.2$ & $2.5 \pm 0.0$ & $0.6 \pm 0.0$ & $25.1 \pm 1.1$ & $34.0 \pm 1.5$ & $30.4 \pm 2.2$ \\
\hline 4 & $11.9 \pm 0.6$ & $10.3 \pm 0.5$ & $2.7 \pm 0.0$ & $0.7 \pm 0.0$ & $28.3 \pm 1.3$ & $36.5 \pm 1.3$ & $26.4 \pm 0.0$ \\
\hline 5 & $15.7 \pm 0.6$ & $10.7 \pm 0.0$ & $2.7 \pm 0.0$ & $0.7 \pm 0.0$ & $33.3 \pm 0.1$ & $56.9 \pm 0.3$ & $45.6 \pm 2.3$ \\
\hline 6 & $17.1 \pm 0.8$ & $10.6 \pm 0.1$ & $2.7 \pm 0.1$ & $0.7 \pm 0.0$ & $36.7 \pm 0.9$ & $60.6 \pm 2.9$ & $56.2 \pm 1.0$ \\
\hline 7 & $20.5 \pm 0.6$ & $11.0 \pm 0.5$ & $2.7 \pm 0.0$ & $0.8 \pm 0.0$ & $37.8 \pm 1.0$ & $68.1 \pm 0.5$ & $60.2 \pm 1.4$ \\
\hline 8 & $22.2 \pm 1.0$ & $10.6 \pm 0.4$ & $2.7 \pm 0.0$ & $0.8 \pm 0.0$ & $46.8 \pm 1.5$ & $72.9 \pm 3.2$ & $65.7 \pm 1.9$ \\
\hline 9 & $23.6 \pm 0.2$ & $10.5 \pm 0.2$ & $2.7 \pm 0.0$ & $0.8 \pm 0.0$ & $48.9 \pm 1.9$ & $85.4 \pm 1.9$ & $63.3 \pm 1.7$ \\
\hline 10 & $24.0 \pm 0.5$ & $11.4 \pm 0.0$ & $2.9 \pm 0.1$ & $0.8 \pm 0.0$ & $46.3 \pm 1.6$ & $69.8 \pm 4.3$ & $77.6 \pm 1.7$ \\
\hline 11 & $24.2 \pm 1.4$ & $10.3 \pm 0.3$ & $2.7 \pm 0.0$ & $0.8 \pm 0.0$ & $53.3 \pm 1.9$ & $90.1 \pm 5.4$ & $69.4 \pm 1.2$ \\
\hline 12 & $25.1 \pm 0.2$ & $10.2 \pm 0.1$ & $2.7 \pm 0.0$ & $0.9 \pm 0.0$ & $57.3 \pm 1.1$ & $94.3 \pm 0.0$ & $67.6 \pm 1.3$ \\
\hline 13 & $25.7 \pm 0.7$ & $11.1 \pm 0.6$ & $2.8 \pm 0.0$ & $0.9 \pm 0.0$ & $54.1 \pm 1.2$ & $112.5 \pm 2.3$ & $70.4 \pm 1.9$ \\
\hline 14 & $26.3 \pm 1.1$ & $11.0 \pm 0.8$ & $2.8 \pm 0.1$ & $0.9 \pm 0.0$ & $57.7 \pm 0.7$ & $102.8 \pm 5.9$ & $76.0 \pm 1.8$ \\
\hline 15 & $26.8 \pm 0.9$ & $11.6 \pm 0.2$ & $2.8 \pm 0.0$ & $0.9 \pm 0.0$ & $61.6 \pm 0.6$ & $113.9 \pm 4.5$ & $80.4 \pm 1.5$ \\
\hline 16 & $26.9 \pm 0.6$ & $11.8 \pm 0.7$ & $2.9 \pm 0.0$ & $0.9 \pm 0.0$ & $60.6 \pm 1.1$ & $109.2 \pm 1.3$ & $76.9 \pm 1.2$ \\
\hline 17 & $26.9 \pm 0.5$ & $11.6 \pm 0.5$ & $2.9 \pm 0.1$ & $1.0 \pm 0.0$ & $62.8 \pm 0.7$ & $111.5 \pm 5.2$ & $82.1 \pm 1.6$ \\
\hline 18 & $28.1 \pm 0.4$ & $11.5 \pm 0.5$ & $2.9 \pm 0.0$ & $1.0 \pm 0.0$ & $72.7 \pm 1.5$ & $115.2 \pm 5.6$ & $85.4 \pm 1.8$ \\
\hline 19 & $23.7 \pm 1.2$ & $10.6 \pm 0.0$ & $2.8 \pm 0.0$ & $0.8 \pm 0.0$ & $51.2 \pm 0.3$ & $86.1 \pm 1.0$ & $67.5 \pm 1.2$ \\
\hline
\end{tabular}

Table 4 Pearson correlation coefficients between amylopectin and amylose structural parameters ${ }^{1}$. AC = amylose content

\begin{tabular}{|c|c|c|c|c|c|c|c|c|c|c|c|c|c|c|c|}
\hline $\begin{array}{l}\text { correlation } \\
\text { coefficients }\end{array}$ & $A C$ & $\beta_{\text {Am, (i) }}$ & $\beta_{\mathrm{Am} \text {,(ii) }}$ & $\beta_{\mathrm{Am}, \text { (iii) }}$ & $h_{\mathrm{Am},(\mathrm{i})}$ & $h_{\mathrm{Am} \text {,(ii) }}$ & $h_{\mathrm{Am}, \text { (iii) }}$ & $\beta_{(i)}$ & $\beta_{(\mathrm{ii})}$ & $\beta_{\text {(iii) }}$ & $\beta_{\text {(iv) }}$ & $\beta_{(v)}$ & $\beta_{(v i)}$ & $h_{(\mathrm{iii} / \mathrm{i})}$ & $h_{(\mathrm{v} / \mathrm{i})}$ \\
\hline$A C$ & 1 & & & & & & & & & & & & & & \\
\hline$\beta_{\mathrm{Am} \text {, (i) }}$ & 0.369 & 1 & & & & & & & & & & & & & \\
\hline$\beta_{\text {Am,(i) }}$ & $0.750^{* *}$ & $0.597^{* *}$ & 1 & & & & & & & & & & & & \\
\hline$\beta_{\text {Am,(iii) }}$ & $0.937^{* *}$ & $0.483^{*}$ & $0.829^{* *}$ & 1 & & & & & & & & & & & \\
\hline$h_{\mathrm{Am} \text {, (i) }}$ & $0.967^{* *}$ & 0.407 & $0.775^{* *}$ & $0.960^{* *}$ & 1 & & & & & & & & & & \\
\hline$h_{\text {Am,(ii) }}$ & $0.963^{* *}$ & 0.417 & $0.721^{* *}$ & $0.938^{* *}$ & $0.965^{* *}$ & 1 & & & & & & & & & \\
\hline$h_{\mathrm{Am}, \text { (iii) }}$ & $0.977^{* *}$ & 0.438 & $0.772^{* *}$ & $0.889^{* *}$ & $0.946^{* *}$ & $0.925^{* *}$ & 1 & & & & & & & & \\
\hline$\beta_{(\mathrm{i})}$ & 0.380 & -0.061 & 0.406 & 0.472 & 0.386 & 0.285 & 0.272 & 1 & & & & & & & \\
\hline$\beta_{\text {(ii) }}$ & -0.246 & 0.204 & -0.046 & $\begin{array}{l}- \\
0.197\end{array}$ & -0.222 & -0.179 & -0.181 & $-0.594^{*}$ & 1 & & & & & & \\
\hline$\beta_{\text {(iii) }}$ & $-0.685^{* *}$ & $\begin{array}{l}- \\
0.318\end{array}$ & -0.376 & $-{ }^{-} .586^{*}$ & $-0.682^{* *}$ & $-\overline{-}^{*} 711^{* *}$ & $\overline{-}^{0.731^{* *}}$ & 0.244 & -0.162 & 1 & & & & & \\
\hline$\beta_{\text {(iv) }}$ & $0.633^{* *}$ & 0.104 & 0.348 & $0.538^{*}$ & $0.560^{*}$ & $0.561^{*}$ & $0.636^{* *}$ & 0.147 & 0.100 & $-0.683^{* *}$ & 1 & & & & \\
\hline$\beta(v)$ & $-0.712^{* *}$ & $\overline{0.321}$ & $-0.550^{*}$ & $-\overline{0.798^{* *}}$ & $\overline{0.697^{* *}}$ & $\overline{0}^{-} 697^{* *}$ & $-\overline{0.615^{* *}}$ & $-0.549^{*}$ & 0.357 & 0.324 & -0.383 & 1 & & & \\
\hline$\beta_{(v i)}$ & 0.123 & -0.079 & -0.094 & 0.120 & 0.061 & 0.159 & 0.049 & 0.052 & -0.033 & 0.087 & 0.018 & -0.252 & 1 & & \\
\hline$h_{(\mathrm{iii} / \mathrm{i})}$ & -0.433 & -0.061 & -0.129 & -0.400 & $-0.477^{*}$ & $-0.534^{*}$ & $\begin{array}{l}- \\
0.391\end{array}$ & -0.053 & 0.313 & 0.398 & -0.192 & 0.451 & -0.063 & 1 & \\
\hline$h_{(\mathrm{V} / \mathrm{i})}$ & -0.139 & -0.120 & 0.008 & -0.217 & -0.202 & -0.196 & $\begin{array}{l}- \\
0.064\end{array}$ & 0.002 & -0.260 & 0.283 & -0.243 & 0.385 & -0.090 & $0.521^{*}$ & 1 \\
\hline
\end{tabular}


biosynthesis (as discussed above). Thus, the parameters in each amylose region are negatively correlated with $\beta_{\text {(iii) }}$ and $\beta_{(\mathrm{v})}$ values. These results might indicate that some elongation of amylose chains happens inside of starch granules, where the synthesis of amylose and amylopectin can compete for substrates and enzymes.

It is found that $\beta_{\text {(iv) }}$ is positively correlated with $\beta_{\mathrm{Am} \text {,(iii), }}$,

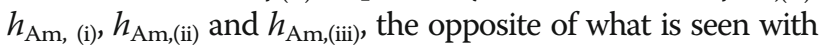
$\beta_{\text {(iii) }}$ and $\beta_{\text {(v) }} \beta_{\text {(iv) }}$ is negatively correlated with $\beta_{\text {(iii), which is }}$ an expected result because the amylopectin model assumes (Wu et al. 2013a) that for rice, the branching enzymes of in these region compete for substrate. Hence this positive correlation is probably a statistical coincidence.

It is seen that $h_{(\text {iii/i) }}$ has a weak negative correlation with $h_{\mathrm{Am} \text {, (i) }}$ and $h_{\mathrm{Am} \text {,(ii) }}$. If this weak correlation is indeed the case, this might be because same SBE isoforms participate in the synthesis of both intermediate amylopectin and short amylose chains. It also could be a statistical coincidence, because it is only a weak correlation and $h_{(\mathrm{iii} / \mathrm{i})}$ does not have a significant correlation with $h_{\mathrm{Am} \text {,(iii), }}$ while the latter is strongly correlated with $h_{\mathrm{Am}}$, (i) and $h_{\mathrm{Am} \text {,(ii). }}$. The internal correlations between amylopectin structural parameters, and internal correlations between amylose structural parameters, are not discussed here as they are not germane to the aim of the present study, which is to look at which enzymes are involved in the synthesis of both amylopectin and amylose.

\section{Conclusions}

Quantitative analysis of the structural parameters for amylopectin and amylose chain length distributions of nineteen natural rice samples indicate that GBSSI competes with SBEI for substrates during starch synthesis in rice. This might be of use for developing rice varieties with desirable amylose contents and CLDs. The results also confirm that GBSSI, SBE and SS are all involved in biosynthesis of short amylose chains.

\section{Methods \\ Materials}

Nineteen natural rice varieties were harvested from the same large field in Sanya, Hainan Province, China; details are listed in Table 1. Dimethyl sulfoxide (DMSO, GR grade for analysis) was purchased from Merck \& Co., Inc. (Kilsyth, VIC, Australia). Protease from Streptomyces griseus (type XIV) and LiBr (ReagentPlus) were purchased from Sigma-Aldrich Pty. Ltd. (Castle Hill, NSW, Australia). Isoamylase (from Pseudomonas sp.) was purchased from Megazyme International, Ltd. (Wicklow, Ireland). 8-Aminopyrene-1,3,6-trisulfonate (APTS), included in the Carbohydrate Labelling and Analysis Kit, was purchased from BeckmanCoulter (Brea, USA). Other chemical reagents were analytical grade and used as received.

\section{Processing of Grains}

Rice grains $(5 \mathrm{~g})$ were precooled in liquid nitrogen for 5 min before being ground to rice flour using a cryogrinder (MM400, Netsch, Germany). Grinding at a low temperature reduces the damage of the molecular and granular structure of starch. All samples were ground for $2 \mathrm{~min}$ at $30 \mathrm{~s}^{-1}$ following the method of (Hasjim et al. 2012).

\section{Preparation of Debranched Samples}

Rice starch was extracted following method of (Syahariza et al. 2010) with some modifications. Rice flour (7-9 mg) was treated by protease in tricine buffer $(0.5 \mathrm{~mL}, 2.5$ Units $/ \mathrm{mL}$ ) in a thermomixer (Thermomixer Comfort, Hanbury, Germany) at $37^{\circ} \mathrm{C}$ for $60 \mathrm{~min}$, then treated with sodium bisulfite solution $(0.5 \mathrm{~mL}, 0.45 \% \mathrm{w} / \mathrm{w})$ at $37^{\circ} \mathrm{C}$ for $30 \mathrm{~min}$. The solution was centrifuged at 4000 rpm for $10 \mathrm{~min}$, and the supernatant containing most of protein was removed. The precipitate was dissolved in $\mathrm{DMSO} / \mathrm{LiBr}$ solution $(1.5 \mathrm{~mL}, 0.5 \% \mathrm{w} / \mathrm{w})$ in a thermomixer at $80^{\circ} \mathrm{C}$ for $24 \mathrm{~h}$, and inverted occasionally by hand to ensure a homogenous mixture. Then the mixture was centrifuged at $4000 \mathrm{rpm}$ for $10 \mathrm{~min}$, and the precipitate (mostly proteins and non-starch polysaccharides) was discarded. Starch was precipitated from the supernatant by adding 5 times the volume of absolute ethanol. The extracted starch was dispersed in boiling deionized water $(0.9 \mathrm{~mL})$ with occasional gentle shaking for at least $1 \mathrm{~h}$. Then it was mixed with sodium azide solution $\left(5 \mu \mathrm{L}, 0.04 \mathrm{~g} \mathrm{~mL}^{-1}\right)$, acetate buffer solution $(0.1$ $\mathrm{mL}, 0.1 \mathrm{M}, \mathrm{pH} 3.5)$ and isoamylase $(2.5 \mu \mathrm{L}, 1000 \mathrm{U} / \mathrm{mL})$, and incubated at $37^{\circ} \mathrm{C}$ for $3 \mathrm{~h}$. The debranching process was stopped by adding $\mathrm{NaOH}$ solution $(0.1 \mathrm{M})$ to adjust the $\mathrm{pH}$ to 7 , and the solution was incubated at $80^{\circ} \mathrm{C}$ for $1 \mathrm{~h}$. All the samples were freeze-dried overnight and stored in a desiccator for SEC and FACE analysis.

\section{SEC Analysis and Amylose Content}

The debranched starch $(\sim 4 \mathrm{mg})$ was dissolved in $1 \mathrm{~mL}$ $\mathrm{DMSO} / \mathrm{LiBr}(0.5 \%, \mathrm{w} / \mathrm{w})$ at $80^{\circ} \mathrm{C}$ overnight, and the solution was transferred into SEC vials for SEC analysis. The SEC separation was carried out with a Shimazu LC20 system (Kyoto, Japan) with a combination of Gram precolumn, Gram 1000 column and Gram 100 column (Polymer Standards Service, Mainz, Germany) in sequence. The eluent used in this study was DMSO-LiBr solution $(0.5 \%, \mathrm{w} / \mathrm{w})$, and the flow rate was set at $0.6 \mathrm{~mL} / \mathrm{min}$. The column temperature was set at $80^{\circ} \mathrm{C}$ and the differential refractive index (DRI) detector temperature was set at $45^{\circ} \mathrm{C}$. The DRI signal was calibrated with a series of pullulan standards ranging from 342 to $2.35 \times 10^{6} \mathrm{Da}$, and these standards provided universal calibration curves to relate elution volume $V_{\text {el }}$ with hydrodynamic volume $V_{\mathrm{h}}$. For a linear polymer such as debranched starch, its $V_{\mathrm{h}}$ can in 
turn be converted to the degree of polymerization (DP the number of monomer units in a chain) $X$ using the Mark-Houwink equation: $V_{\mathrm{h}}=\frac{2}{5} \frac{K\left(X M_{0}+18\right)^{1+\alpha}}{N_{\mathrm{A}}}: M_{0}=16$ 2.2 is the molecular weight of the anhydroglucose monomer unit and 18 is that of the additional water in the end groups, $N_{\mathrm{A}}$ is Avogadro's constant, $\mathrm{K}$ and $\alpha$ for linear starch chains in the eluent of $\mathrm{DMSO} / \mathrm{LiBr}$ at $80^{\circ} \mathrm{C}$ are $1.5 \times 10^{-4} \mathrm{dL} \mathrm{g-1}$ and 0.743 , respectively (Vilaplana and Gilbert 2010a; Liu et al. 2010). The result is the SEC weight distribution $w(\log X)$. Although of course SEC signals vary with elution times in different runs, such variation is explicitly taken into account by the SEC calibration used here; that is why one must never present SEC data as a function of elution volumes (Gidley et al. 2010), which are instrumental quantities, but instead be presented as functions of molecular properties such as $X$ and $R_{\mathrm{h}}$.

The amylose content was found by calculating the ratio of the area under the curve (AUC) of amylose composition (at the value of $X$ where there is a clear division between short amylopectin chains and long amylose chains, at $X \sim 100)$ to the AUC of the entire starch composition (Vilaplana et al. 2012).

The value of the amylose content depends on the method of measurement (Gray-Weale and Gilbert 2009), and is often therefore denoted "apparent amylose content". Separating the amylose and amylopectin chains at the DP $(\sim 100)$ where there is a minimum, as we do here, is a simplification, as it ignores contributions to amylopectin chains by very short amylose ones, and to amylose chains by very long amylopectin ones. All 19 rice samples we used in this experiment have moderate amylose content (the highest is $28.1 \%$ ), and they all show normal debranched starch distributions. While there can be some extra-long amylopectin chains, starch with a significant number of such chains is high-amylose starch (e.g. (Li et al. 2019)), and their debranched distributions are very different from normal starch. Thus the samples used in this study unlikely have very long amylopectin chains, and the amylose content calculated from the overall CLD is close to true amylose content. For high-amylose starches, the whole question of defining amylose content becomes much more complex, and can really only be performed using two-dimensional analysis (Vilaplana and Gilbert 2010b; Vilaplana et al. 2012; Vilaplana et al. 2014), where one dimension is the length of individual chains, and the other is the total molecular size.

\section{Amylopectin CLDs}

The CLDs of the amylopectin component were analyzed using FACE. Debranched starch $(0.2 \mathrm{mg})$ was labeled with $1.5 \mu \mathrm{L}$ APTS solution (5 mg of APTS in
$50 \mu \mathrm{L}$ of $15 \%$ glacial acetic acid) and $1.5 \mu \mathrm{L}$ sodium cynoborohydride, and incubated in a thermomixer at $60{ }^{\circ} \mathrm{C}$ for $4 \mathrm{~h}$ in the dark. Then $80 \mu \mathrm{L}$ deionized water was added to the sample, and the solution was vortexed at a low speed to until dissolution was complete. $50 \mu \mathrm{L}$ of the solution was transferred to FACE micro-vials for analysis, using a P/ACE MDQ plus system (Ab Sciex, US). The analysis was conducted following the method of (Bai et al. 2017). FACE directly gives the number CLD, $N_{\text {de }}(X)$, the number distribution of chains containing $X$ monomer units after debranching. The relation between the number and weight distributions is $w(\log X)=X^{2}$ $N_{\text {de }}(X)$ (Castro et al. 2005).

The $N_{\mathrm{de}}(X)$ were fitted with the Wu-Gilbert model (Wu and Gilbert 2010; Wu et al. 2013a). The model assumes that the CLD is controlled by isoforms of the three types of starch biosynthetic enzymes, SS, SBE and DBE. It also assumes that different "enzyme sets", comprising one isoform of each type, contribute to the overall CLD, with different sets being major, but not exclusive, sources of chains for different ranges of DP. The rather complex equations quantifying the CLD are expressed in terms of two parameters for each set: $\beta_{i}$ and $h_{i}$, being respectively the ratio of the activity of SBE to that of SS in set $i$, and the relative contribution of that set to the overall CLD; in some regions, two sets can compete for the same substrates. For reasons discussed elsewhere (Wu et al. 2013a), the fit to the accurate CLD from FACE involves the parameters $\beta_{(\mathrm{i})}, \beta_{(\mathrm{ii})}, \beta_{(\mathrm{iii})}, \beta_{(\mathrm{iv})}$, $\beta_{(\mathrm{v})}, \beta_{(\mathrm{vi})}, h_{(\mathrm{iii} / \mathrm{i})}$ and $h_{(\mathrm{v} / \mathrm{i})}$. The fit uses publicly available code (Wu et al. 2013a).

\section{Amylose CLDs}

The CLDs of amylose were fitted to a different mathematical model with similar assumptions, but this time implicitly involving GBSS as well (Nada et al. 2017; Yu et al. 2019). The treatment takes partial account of SEC band broadening. The amylose parameters for the $i^{\text {th }}$ set are denoted $\beta_{\mathrm{Am}, i}$ and $h_{\mathrm{Am}, i .}$. In this study, three different features in the amylose CLD can be distinguished, giving 6 parameters for the fitting: $\beta_{\mathrm{Am} \text {, (i) }}, \beta_{\mathrm{Am} \text {,(ii) }}, \beta_{\mathrm{Am} \text {,(iii) }}, h_{\mathrm{Am}}$, (i), $h_{\mathrm{Am} \text {,(ii) }}$ and $h_{\mathrm{Am} \text {,(iii). Again the fitting was performed }}$ with publicly available code (Nada et al. 2017).

\section{Statistical Analysis}

The correlation of amylopectin and amylose molecular structural parameters were analyzed with Pearson correlation analyses using IBM SPSS, to reveal the possible connections between amylose biosynthesis and amylopectin biosynthesis. Statistical significance was set at a probability level of 0.05 . 


\section{Supplementary information}

Supplementary information accompanies this paper at https://doi.org/10. 1186/s12284-019-0353-3

Additional file 1: Figure S1-a. Experiment ("exp", from FACE) and model-fitted ("cal") number chain-length distributions $\left(N_{\text {de }}(X)\right.$, arbitrary normalization) of debranched amylopectin (DP < 100) from sample 1 to 9. Figure S1-b. Experiment ("exp", from FACE) and model-fitted ("cal") number chain-length distributions ( $N_{\text {de }}(X)$, arbitrary normalization) of debranched amylopectin (DP < 100) from samples 10 to 19. Figure S2-a. Experiment ("exp", from SEC) and model-fitted ("fit") weight chain-length distributions ( $w(\log X)$, arbitrary normalization) of debranched amylose (DP > 100) from samples 2 to 10. Figure S2-b. Experiment ("exp", from SEC) and model-fitted ("fit") weight chain-length distributions ( $w(\log X)$, arbitrary normalization) of debranched amylose (DP > 100) from samples 11 to 19.

\section{Abbreviations}

APTS: 8-Aminopyrene-1,3,6-trisulfonic acid, trisodium salt; CLD: Chain length distribution; DBE: debranching enzyme; DP: Degree of polymerization; FACE: Fluorophore-assisted carbohydrate electrophoresis; GBSS: Granulebound starch synthase; SBE: Starch branching enzyme; SEC: Size exclusion chromatography; SS: Starch synthase

\section{Acknowledgements}

We thank Mr. Shiqing Zhou (Polysaccharide Research Centre, Yangzhou, China) for SEC and FACE data processing, We thank Prof. Qiaoquan Liu and Dr. Changquan Zhang for their scientific knowledge support.

\section{Authors' Contributions}

RGG conceived the overall approach of the work, EL and HH drafted the manuscript, $\mathrm{HH}, \mathrm{CY}$ and EL designed the experiment, JZ and LZ provided all the rice samples, and $Y B, H H$, EL and RGG edited the manuscript. All authors read and approved the final manuscript.

\section{Authors' Information}

$\mathrm{HH}$ is a MS student in Yangzhou University (China), CY is a bachelor student in Yangzhou University (China), JZ is a Ph. D candidates in Yangzhou University (China), LZ is a research fellow in Shanghai Academy of Agricultural Sciences (China), EL is a lecturer in Yangzhou University, YB is a PhD student at the University of Queensland, and RGG is a professor in both Yangzhou University (China) and the University of Queensland (Australia).

\section{Funding}

We gratefully acknowledge the support of a Jiangsu provincial Entrepreneurial and Innovation Phd Program, and a Yangzhou Lvyangjinfeng Talent Program (to EL), a National Natural Science Foundation of China grant (C1304013151101138, to both RGG and EL), the Priority Academic Program Development of Jiangsu Higher Education Institutions (EL and RGG), a Jiangsu Entrepreneurship and Innovation Team program (to R.G.G.), and a Shanghai Agriculture Applied Technology Development Program, China (Grant No.G2016060101, to LZ). Youth Fund of Jiangsu Natural Science Foundation (BK20190906, to EL), and Jiangsu Yangzhou Key Research and Development Program (No. SSF2018000008, to EL).

\section{Availability of Data and Materials}

All data generated or analysed during this study are included in this published article and its supplementary information files.

\section{Ethics Approval and Consent to Participate}

Not applicable.

\section{Consent for Publication}

Not applicable.

\section{Competing Interests}

All the authors declare that they have no competing interests.

\section{Author details}

1 Jiangsu Key Laboratory of Crop Genetics and Physiology, Key Laboratory of Plant Functional Genomics of the Ministry of Education, Jiangsu Key
Laboratory of Crop Genetics and Physiology, College of Agriculture, Yangzhou University, Yangzhou 225009, People's Republic of China. ${ }^{2}$ Co-Innovation Center for Modern Production Technology of Grain Crops, Yangzhou University, Yangzhou 225009, China. ${ }^{3}$ Crop Breeding and Cultivation Research Institute, Shanghai Academy of Agricultural Sciences, Shanghai 201403, China. ${ }^{4}$ Centre for Nutrition \& Food Sciences, Queensland Alliance for Agriculture \& Food Innovations, The University of Queensland, QLD, Brisbane 4072, Australia.

Received: 13 July 2019 Accepted: 29 November 2019

Published online: 23 December 2019

\section{References}

Akihiro T, Mizuno K, Fujimura T (2005) Gene expression of ADP-glucose Pyrophosphorylase and starch contents in Rice cultured cells are cooperatively regulated by sucrose and ABA. Plant Cell Physiol 46(6):937-946. https://doi.org/10.1093/pcp/pci101

Bai Y, Wu P, Wang K, Li E, Gilbert RG (2017) Effects of pectin on molecular structural changes in starch during digestion. Food Hydrocoll 69(1):10-18. https://doi.org/10.1016/j.foodhyd.2017.01.021

Ball SG, van de Wal MHBJ, Visser RGF (1998) Progress in understanding the biosynthesis of amylose. Trends Plant Sci 3(12):462-467. https://doi.org/10. 1016/S1360-1385(98)01342-9

Baroja-Fernandez E, Jose Munoz F, Bahaji A, Almagro G, Pozueta-Romero J (2012) Reply to Smith et al:: no evidence to challenge the current paradigm on starch and cellulose biosynthesis involving sucrose synthase activity. PNAS 109(14):E777-E777

Bowsher CG, Scrase-Field EFAL, Esposito S, Emes MJ, Tetlow IJ (2007) Characterization of ADP-glucose transport across the cereal endosperm amyloplast envelope. J Exp Bot 58:1321-1332. https://doi.org/10.1093/jxb/erl297

Buleon A, Colonna P, Planchot V, Ball S (1998) Starch granules: structure and biosynthesis. Int J Biological Macromolecules 23 (2):85-112

Castro JV, Ward RM, Gilbert RG, Fitzgerald MA (2005) Measurement of the molecular weight distribution of debranched starch. Biomacromolecules 6(4): 2260-2270. https://doi.org/10.1021/bm050041t

Chiaramonte E, Rhazi L, Aussenac T, White DR (2012) Amylose and amylopectin in starch by asymmetric flow field-flow fractionation with multi-angle light scattering and refractive index detection (AF4-MALS-RI). J Cereal Sci 56(2): 457-463. https://doi.org/10.1016/j.jcs.2012.04.006

Denyer K, Johnson P, Zeeman S, Smith AM (2001) The control of amylose synthesis. J Plant Physiol 158:479-487

Gidley MJ, Hanashiro I, Hani NM, Hill SE, Huber A, Jane J-L, Liu Q, Morris GA, Rolland-Sabaté A, Striegel A, Gilbert RG (2010) Reliable measurements of the size distributions of starch molecules in solution: current dilemmas and recommendations. Carbohydr Polym 79(2):255-261. https://doi.org/10.1016/j. carbpol.2009.07.056

Gray-Weale A, Gilbert RG (2009) General description of the structure of branched polymers. J Polym Sci Part A Polymer Chem Ed 47(15):3914-3930. https://doi. org/10.1002/pola.23458

Guan HP, Preiss J (1993) Differentiation of the properties of the branching isozymes from maize (Zea mays). Plant Physiol 102(4):1269-1273

Hasjim J, Li E, Dhital S (2012) Milling of rice grains: the roles of starch structures in the solubility and swelling properties of rice flour. Starch-Staerke 64(8): 631-645

Hennen-Bierwagen TA, Liu F, Marsh RS, Kim S, Gan Q, Tetlow IJ, Emes MJ, James MG, Myers AM (2008) Starch biosynthetic enzymes from developing maize endosperm associate in multisubunit complexes. Plant Physiol 146(4):1892-1908

Li C, Powell PO, Gilbert RG (2017) Recent progress toward understanding the role of starch biosynthetic enzymes in the cereal endosperm. Amylase 1:59-74. https://doi.org/10.1515/amylase-2017-0006

Li E, Wu AC, Li J, Liu Q, Gilbert RG (2015) Improved understanding of rice amylose biosynthesis from advanced starch structural characterization. Rice 8(1):20. https://doi.org/10.1186/s12284-015-0055-4

Li H, Yu W, Slade AJ, Dhital S, Gidley MJ, Gilbert RG (2019) Starch-branching enzymes contributing to amylose and amylopectin fine structure in wheat submitted

Liu W-C, Halley PJ, Gilbert RG (2010) Mechanism of degradation of starch, a highly branched polymer, during extrusion. Macromolecules 43(6):2855-2864 https://doi.org/10.1021/ma100067x 
Nada SS, Zou W, Li C, Gilbert RG (2017) Parameterizing amylose chain-length distributions for biosynthesis-structure-property relations. Analytical \& Bioanalytical Chemistry 409(29):6813-6819. https://doi.org/10.1007/s00216017-0639-5

Nakamura Y, Kubo A, Shimamune T, Matsud T, Harada K, Satoh H (1997) Correlation between activities of starch debranching enzyme and alphapolyglucan structure in endosperms of sugary-1 mutants of rice. Plant J 12(1):143-153

Satoh H, Nishi A, Yamashita K, Takemoto Y, Tanaka Y, Hosaka Y, Sakurai A, Fujita N, Nakamura Y (2003) Starch-branching enzyme I-deficient mutation specifically affects the structure and properties of starch in Rice endosperm. Plant Physiol 133:1111-1121

Syahariza ZA, Li E, Hasjim J (2010) Extraction and dissolution of starch from cereal grains for accurate structural analysis. Carbohydr Polym 82(1):14-20

Tanaka N, Fujita N, Nishi A, Satoh H, Hosaka Y, Ugaki M, Kawasaki S, Nakamura Y (2004) The structure of starch can be manipulated by changing the expression levels of starch branching enzyme $\mathrm{llb}$ in rice endosperm. Plant Biotechnol J 2(6):507-516

Tester RF, Karkalas J, Qi X (2004) Starch-composition, fine structure and architecture. J Cereal Sci 39(2):151-165

Vilaplana F, Gilbert RG (2010a) Characterization of branched polysaccharides using multiple-detection size separation techniques. J Sep Sci 33(22):35373554. https://doi.org/10.1002/jssc.201000525

Vilaplana F, Gilbert RG (2010b) Two-dimensional size/branch length distributions of a branched polymer. Macromolecules 43(17):7321-7329. https://doi.org/10. 1021/ma101349t

Vilaplana F, Hasjim J, Gilbert RG (2012) Amylose content in starches: towards optimal definition and validating experimental methods. Carbohydr Polym 88(1):103-111. https://doi.org/10.1016/j.carbpol.2011.11.072

Vilaplana F, Meng D, Hasjim J, Gilbert RG (2014) Two-dimensional. macromolecular distributions reveal detailed architectural features in highamylose starches. Carbohydr Polym 113(1):539-551. https://doi.org/10.1016/j. carbpol.2014.07.050

Wang K, Hasjim J, Wu AC, Li E, Henry RJ, Gilbert RG (2015) Roles of GBSSI and SSIla in determining amylose fine structure. Carbohydr Polym 17(1):264-274. https://doi.org/10.1016/j.carbpol.2015.03.081

Wu AC, Gilbert RG (2010) Molecular weight distributions of starch branches reveal genetic constraints on biosynthesis. Biomacromolecules 11(12):35393547. https://doi.org/10.1021/bm1010189

Wu AC, Morell MK, Gilbert RG (2013a) A parameterized model of amylopectin synthesis provides key insights into the synthesis of granular starch. PLoS One 8(6):e65768. https://doi.org/10.1371/journal.pone.0065768

Wu AC, Witt T, Gilbert RG (2013b) Characterization methods for starch-based materials: state of the art and perspectives. Aust J Chem 66(12):1550-1563. https://doi.org/10.1071/CH13397

Yu W, Li H, Zou W, Tao K, Zhu J, Gilbert RG (2019) Using starch molecular fine structure to understand biosynthesis-structure-property relations. Trends in Food Science \& Technology 86:530-536. https://doi.org/10.1016/j.tifs.2018.08.003

Yu W, Tao K, Gilbert RG (2018) Improved methodology for analyzing relations between starch digestion kinetics and molecular structure. Food Chem 264: 284-292. https://doi.org/10.1016/j.foodchem.2018.05.049

Zeeman SC, Kossmann J, Smith AM (2010) Starch: its metabolism, evolution, and biotechnological modification in plants. In: Annual review of plant biology, vol 61. Annual Reviews, Palo Alto, pp 209-234. https://doi.org/10.1146/ annurev-arplant-042809-112301

Zhang X, Szydlowski N, Delvalle D, D'Hulst C, James MG, Myers AM (2008) Overlapping functions of the starch synthases SSII and SSIII in amylopectin biosynthesis in Arabidopsis. BMC Plant Biol 8:18

\section{Publisher's Note}

Springer Nature remains neutral with regard to jurisdictional claims in published maps and institutional affiliations.

\section{Submit your manuscript to a SpringerOpen ${ }^{\circ}$ journal and benefit from:}

- Convenient online submission

- Rigorous peer review

- Open access: articles freely available online

- High visibility within the field

- Retaining the copyright to your article

Submit your next manuscript at $\boldsymbol{\nabla}$ springeropen.com 